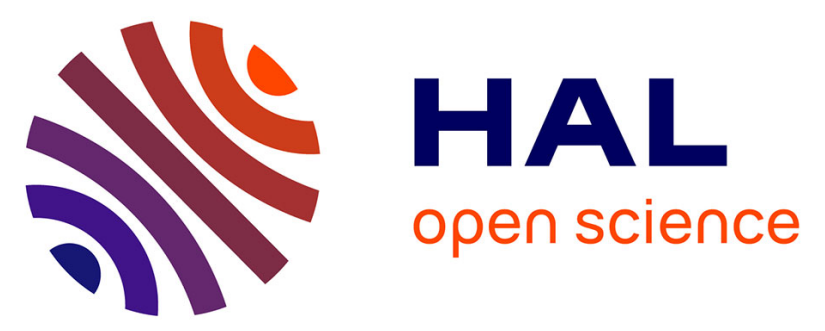

\title{
En-deçà de l'au-delà : le zombie aux limites de la fiction. Les effets de réel dans les romans de Max Brooks et la série télévisée The Walking Dead
}

Clémentine Hougue

\section{- To cite this version:}

Clémentine Hougue. En-deçà de l'au-delà : le zombie aux limites de la fiction. Les effets de réel dans les romans de Max Brooks et la série télévisée The Walking Dead. Trans: Revue de Littérature Générale et Comparée, 2014, 10.4000/trans.1017 . hal-02443718

\section{HAL Id: hal-02443718 \\ https://hal.science/hal-02443718}

Submitted on 17 Jan 2020

HAL is a multi-disciplinary open access archive for the deposit and dissemination of scientific research documents, whether they are published or not. The documents may come from teaching and research institutions in France or abroad, or from public or private research centers.
L'archive ouverte pluridisciplinaire HAL, est destinée au dépôt et à la diffusion de documents scientifiques de niveau recherche, publiés ou non, émanant des établissements d'enseignement et de recherche français ou étrangers, des laboratoires publics ou privés. 


\section{TRANS-}

Revue de littérature générale et comparée

17 | 2014

Au-delà

\section{En-deçà de l'au-delà : le zombie aux limites de la fiction. Les effets de réel dans les romans de Max Brooks et la série télévisée The Walking Dead}

\section{Clémentine Hougue}

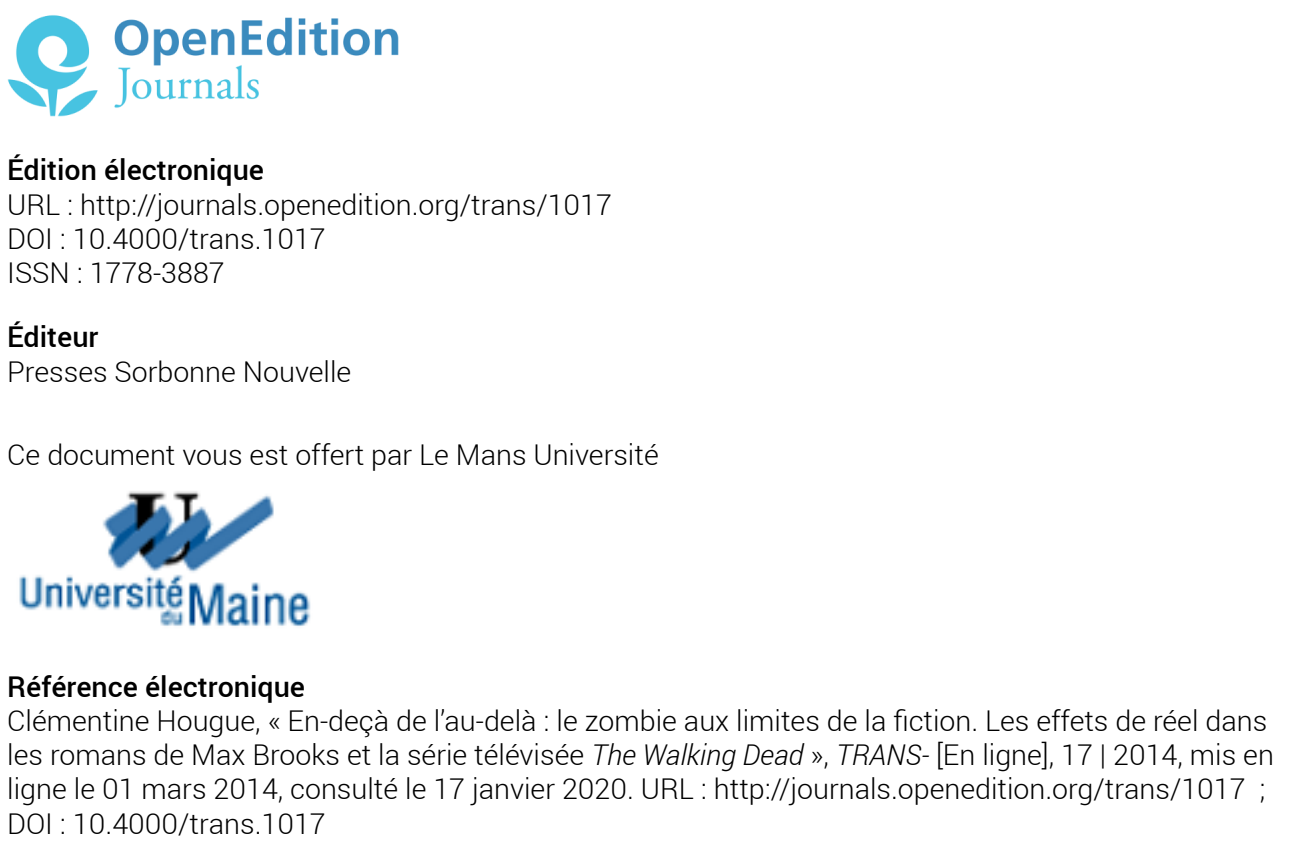

Ce document a été généré automatiquement le 17 janvier 2020.

Tous droits réservés 


\title{
En-deçà de l'au-delà : le zombie aux limites de la fiction. Les effets de réel dans les romans de Max Brooks et la série télévisée The Walking Dead
}

\author{
Clémentine Hougue
}

1 Issu d'une « tradition à la fois orale et extra-européenne ${ }^{1}$ », celle du vaudou haïtien, où il désigne un individu plongé dans un état de catalepsie par l'usage de drogues, le zombie (ou zombi dans son orthographe haïtienne), investit le champ cinématographique dès les années trente pour devenir archétypal dans les films de George A. Romero. Le zombie, ou mort-vivant, cadavre en décomposition, animé de mouvements le plus souvent lents et maladroits, est une figure désormais traditionnelle du film gore. Oxymore incarné, le mort-vivant effraie par son inclassabilité : par définition en-deçà de l'au-delà, il se trouve dans un espace-limite, encore en-deçà de la mort mais déjà au-delà de la vie.

2 Longtemps cantonné à la série $\mathrm{Z}$, le « film de zombie » tend graduellement à devenir un genre à part entière et en conséquence gagne en reconnaissance et en popularité. Depuis le début des années 2000, la figure du zombie s'hybride avec d'autres genres, la comédie sociale (Shaun of the Dead) ou le teen-movie (Zombieland), pour investir celui de la série télévisée : The Walking Dead, créée en 2010 par Franck Darabont et Robert Kirkman, est une adaptation télévisuelle de la bande dessinée du même nom, scénarisée depuis 2003 par le même Robert Kirkman et dessinée successivement par Tony Moore (pour les six premiers chapitres) puis par Charlie Adlard. L'intrigue de The Walking Dead commence après une mystérieuse invasion de zombie qui a rendu le monde entier à l'état sauvage ; dans ce contexte privé d'état, d'infrastructures, d'économie, un groupe d'individus tente de reconstruire une communauté pour survivre. Rick, ancien policier, sa femme Lori, leur fils Carl et Shane, ami et collègue de Rick et brièvement amant de Lori lorsque celle-ci a cru son époux mort, forment le noyau dur de ce groupe autour duquel gravite un grand nombre de personnages. 
3 Hormis le célèbre I am legend de Robert Matheson (1954), le roman n'est pas la forme fictionnelle la plus courante pour développer le motif du zombie; or, depuis les années 2000, Max Brooks a investit ce champ grâce à deux romans qui proposent des structures narratives inhabituelles: The Zombie Survival Guide (2003), traduit en français sous le titre Guide de survie en territoire zombie, qui se présente comme un guide pratique de lutte contre les zombies et World War Z (2006), qui se présente sous la forme d'une collection d'interviews réalisées par un ancien émissaire de l'ONU après que la guerre contre les zombies a eu lieu. Les témoignages, de civils comme de militaires, d'anonymes et de responsables, retracent les différentes étapes de cette invasion.

4 La figure du zombie, en tant que construction historique, manifeste le fantasme d'une eschatologie où les instruments de la destruction du monde seraient ces personnages hybrides, à la fois morts et vivants ; dans les textes de Max Brooks et la série The Walking Dead, on verra que cette figure devient l'outil d'une déconstruction des rapports entre réel et fiction. Nous nous proposons ainsi de montrer comment le zombie, fiction de la fin du monde, explore paradoxalement les limites de la fiction et de la réalité.

\section{Nature et fonctions du zombie : fantasme et fiction de la fin du monde}

$5 \quad$ Avant de s'intéresser aux cas particuliers que représentent les œuvres de Max Brooks et la série The Walking Dead, il parait nécessaire de comprendre ce que la figure du mortvivant représente et ce qui, sur le plan historique et culturel, s'y incarne. En effet, le zombie apparaît d'emblée comme un personnage à la fois inclassable et symptomatique des angoisses de l'Occident moderne : s'il peut incarner la crainte d'un progrès technique, dont le développement irraisonné conduirait à la création monstrueuse d'hybrides échappant au contrôle humain, le zombie se présente également comme le fantasme d'une punition divine, le désir d'une Apocalypse qui renverserait les lois de la nature. Maxime Coulombe, dans sa Petite philosophie du zombie, explique ainsi que « le cinéma de zombies ressasse un souhait [...] : une part de nous rêve d'assister à la fin du monde ${ }^{2}$ ». Ainsi, selon le philosophe, la figure du mort-vivant et son succès grandissant depuis la fin des années soixante révèlent "une certaine fatigue, une volonté d'en finir et un doute grandissant sur la singularité de l'homme, sur le sens de la civilisation occidentale ${ }^{3}$ ".

6 À la différence d'autres monstres modernes issus de transformations humaines (comme le vampire, par exemple), le mort-vivant est sans conscience, sans affect et sans instinct de survie ; seuls ses mouvements caractéristiques le distinguent d'un cadavre. Il n'a donc pas de volonté, ni de vivre, ni de nuire, ni de tuer : la destruction qu'il porte dans son sillage est dépourvue de toute intention. Dès lors, le mort-vivant réaliserait le fantasme d'une tabula rasa civilisationnelle reposant sur un être qui serait à la fois la victime du déclin de l'homme (le zombie n'est rien d'autre qu'un humain mort revenu à la vie) et bras armé de la destruction de la société industrielle.

7 Car si le zombie incarne la duplicité de notre rapport à la fin, entre crainte et désir, il est lui-même un être double, non seulement parce qu'il occupe cet espace-limite entre vie et mort, mais aussi parce qu'il se présente, selon Jean-Baptiste Thoret, comme «à la fois ressemblant et radicalement autre. Le zombie n'est donc pas un être nouveau mais une créature grosse de son passé humain ${ }^{4} »$. Aussi le zombie voit-il sa radicale altérité imprégnée des résidus de son humanité passée : il se révèle alors profondément ambigu, 
car il se donne à voir simultanément comme antithèse et comme prolongement de l'homme.

8 C'est certainement sur ces zones d'ambigüité (entre mort et vivant, humain et nonhumain, symptôme et remède, crainte et désir) que le mort-vivant a construit son histoire et a acquis le succès que l'on connaît. Il est ainsi devenu une des plus récentes et des plus populaires fictions contemporaines, fiction entendue au sens d'une modélisation du monde. Il catalyse tout autant des angoisses que des fantasmes, qu'il s'agisse de vie après la mort ou de chute de la civilisation ; il est, en tant qu'aberration (l'impossible état d'un être à la fois mort et vivant), une pure fiction, l'incarnation fantasmatique de peurs et de désirs occidentaux. Mais comme l'écrit Jean-Marie Schaeffer dans Pourquoi la fiction ? : « La question primordiale n'est pas celle des relations que la fiction entretient avec la réalité ; il s'agit plutôt de voir comment elle opère dans la réalité, c'est-à-dire dans nos vies ${ }^{5}$ ». Il se trouve que cette question essentielle se trouve mise en exergue par les romans de Max Brooks et la série de Franck Darabont : le traitement qu'ils proposent est en effet non seulement une nouvelle approche de la figure du zombie, mais aussi, et peut-être surtout, à travers la récurrence des effets de réel que ces œuvres mettent en place, un questionnement en actes de la fiction, ses modalités et ses potentialités.

\section{Zombies et effets de réel : fiction de la fin, limites de la fiction} réel ", paru dans la revue Communications en 1968. Barthes s'attache à définir ce qui se donne pour réel dans le roman réaliste français :

C'est là ce qu'on pourrait appeler l'illusion référentielle. La vérité de cette illusion est celle-ci : supprimé de l'énonciation réaliste à titre de signifié de dénotation, le « réel » y revient à titre de signifié de connotation; car dans le moment où ces détails sont réputés dénoter directement le réel, ils ne font rien d'autre, sans le dire, que le signifier : le baromètre de Flaubert, la petite porte de Michelet ne disent finalement rien d'autre que ceci : nous sommes le réel; c'est la catégorie du "réel» (et non ses contenus contingents) qui est alors signifiée ; autrement dit, la carence même du signifié au profit du seul référent devient le signifiant même du réalisme : il se produit un effet de réel, fondement de ce vraisemblable inavoué qui forme l'esthétique de toutes les œuvres courantes de la modernité. ${ }^{6}$

10 C'est ainsi le détail qui fait effet de réel dans le roman réaliste, c'est-à-dire que c'est l'insignifiant qui se met à signifier. Si cette définition de l'effet de réel s'inscrit dans le cadre du roman réaliste, elle se trouve encore opérante dans le roman de science-fiction ; toutefois, elle peut et, concernant nos objets, doit être élargie : l'effet de réel est toujours ce détail (souvent en lien avec une expérience quotidienne du lecteur) qui «signifie le réel », mais il est aussi ce qui rattache profondément le monde fictionnel au nôtre, à savoir un ensemble de référents communs au réel et à la fiction, notamment des référents socio-historiques. L'effet de réel opèrerait ainsi plus globalement dans tous les indices, procédures et jeux référentiels qui fragilisent l'unité et l'autonomie de la fiction. Richard Saint-Gelais, «L'effet de non-fiction : fragments d'une enquête » nomment ces effet des

indices de non-fictionnalité : lieux textuels où le texte prétend abdiquer ses droits à la construction imaginaire, sorties de la fiction ménagées de 
l'intérieur même de cette fiction, garde-fous faits de mots et, du coup, pris dans toutes les manœuvres déstabilisantes que le discours peut mener à leur endroit.? entre le texte et des référents réels. Il suffit de constater que tout texte de fiction inclut des éléments encyclopédiques reconnaissables ${ }^{8} »$. Ce qui nous intéresse dans le cas des romans de Brooks et dans la série The Walking Dead, c'est la manière dont ces «éléments encyclopédiques reconnaissables" s'inscrivent dans des univers science-fictionnels mettant en scène l'irreprésentable par excellence : le mort-vivant.

\section{Indices de non-fictionnalité dans les œuvres de Max Brooks}

Les effets de réel (qu'il s'agisse du détail barthien, de l'effet de non-fiction modélisé par Richard Saint-Gelais ou du rapport spécifique de la série télévisée au réel, que nous étudierons plus loin) présents dans la plupart des romans ou séries télévisées se configurent ainsi de manière singulière lorsqu'il s'agit de zombies : en effet, l'émergence d'effets de réel crée un rapport paradoxal entre le motif fictionnel du zombie - figurant un état à la fois inenvisageable et fantasmatique d'un être dans le même temps mort et vivant - et une structure fictionnelle poreuse au réel, qui laisse ainsi cet invraisemblable zombie s'immiscer dans le réel via les fissures que créent les indices de non-fictionnalité.

Chez Max Brooks, c'est en franchissant la frontière du fictionnel et du documentaire que se construit l'effet de réel: en effet, son Zombie Survival Guide se structure comme un véritable guide pratique, donnant au lecteur, auquel il s'adresse directement, quantité de détails concrets sur la conduite à tenir en cas d'invasion de zombies. Max Brooks sort ainsi du cadre de la science-fiction, et plus encore du cadre de la fiction, en faisant de son texte un écrit à visée pragmatique. L'auteur explique ainsi:

\footnotetext{
I wrote The Zombie Survival Guide as a real guidebook. Take out the zombies and it's How To Survive A Disaster. The inspiration for The Zombie Survival Guide was growing up in Southern California in the 80s with threats of earthquake and nuclear war. Then in the 90s, we had riots, we had fires, we had floods. In LA we're always living on the edge of disaster [... $]^{9}$

J'ai écrit Guide de survie en territoire zombie comme un véritable guide pratique. Enlevez les zombies et vous obtenez Comment survivre à un désastre. Guide de survie en territoire zombie s'inspire des menaces de tremblements de terre et de guerre nucléaire dans les années 80 en Californie du Sud. Puis dans les années 90, nous avons connu des émeutes, des incendies, des inondations. À Los Angeles nous vivons en permanence au bord du désastre $[\ldots]$
}

La forme de la non-fiction instaure ainsi une hésitation concernant la nature du texte: l'énonciation de The Zombie Survival Book, reposant sur l'adresse au lecteur, maintient un contact permanent avec ce dernier. Les "éléments encyclopédiques reconnaissables" sont multipliés, à travers notamment une liste exhaustive des armes réelles (du manche de pioche à l'AK-47, en passant par l'acide et les radiations) qu'il est possible d'utiliser face à un zombie, et l'évaluation méthodique de leur efficacité. Ainsi, dans le livre de Max Brooks, l'effet de réel devient le moteur même de la fiction : l'approche pragmatique du Guide devient le point d'entrée du lecteur dans la fabula. En introduisant une ambigüité quant à la dimension fictionnelle du texte, Max Brooks s'assure l'adhésion d'un lecteur 
qui, se trouvant en permanence mobilisé, accepte d'autant mieux de prendre pour vrais les évènements relatés.

Si The Zombie Survival Guide revêt une dimension satirique (la liste des armes et de leur efficacité contre les zombies n'est rien moins qu'une critique ironique de la passion des Nord-Américains pour les armes à feu), le deuxième opus de Max Brooks, World War Z, présente à première vue une approche plus traditionnelle. Il s'agit d'une compilation d'interviews, offrant une myriade de points de vue individuels sur la guerre contre les zombies. L'effet de réel est tout d'abord ici porté par la multiplication des personnages, témoins relatant une expérience singulière. La diégèse s'organise donc sur le morcellement des évènements au gré de la parole de ces témoins: au lecteur de reconstruire le puzzle. La multiplication des points de vue individuels fonctionne moins comme une addition ou un empilement des subjectivités que comme une mise en réseau de celles-ci, autrement dit la création d'une intersubjectivité. L'intersubjectivité qui préside à la construction narrative de World War $Z$ déconstruit à la fois la relativité des subjectivités individuelles et l'absolutisme d'une objectivité qui pourrait être simulée par un narrateur omniscient. Husserl faisait de l'intersubjectivité, en tant que relation réciproque des consciences, l'origine et la condition d'un monde commun; de même, la narration intersubjective de World War $Z$ crée ce monde commun au lecteur et aux personnages dont l'irréductible extériorité (ils n'existent pas en dehors de l'interview et ne sont pas - ou très peu - décrits par le narrateur) devient un indice de nonfictionnalité.

16 L'effet de réel repose également sur le fait que sont évoqués à de nombreuses reprises des évènements historiques réels : l'univers dans lequel s'inscrit la diégèse de World War $Z$, quoique futuriste, reste un univers socio-historiquement comparable au nôtre. Ce n'est pas là une réelle innovation : le Londres désert et parfaitement silencieux qui apparaît au début du film de Danny Boyle 28 days later ${ }^{10}$ procède de cette identification à un univers commun. Mais combiné à la forme de l'interview, l'espace géographique et historique de la fiction se superpose d'autant mieux à la réalité. De plus, les interventions des personnages abordent des problématiques bien réelles, ce qui renforce la proximité de l'espace fictionnel avec notre vécu socio-historique. Par exemple, le personnage de Travis d'Ambrosia, vieux général interrogé sur la gestion des premières crises zombies, déclare : "Vous savez combien ça coûte de donner un uniforme à un jeune citoyen américain ? Et je ne parle pas du temps qu'il passe effectivement dans cet uniforme, non, mais de l'entrânement, de l'équipement, de la nourriture, du logement, du transport, des soins médicaux.... ${ }^{11}$ ( ( Do you know the price tag of putting just one American citizen in uniform? And I don't just mean the time that he's actively in that uniform: the training, the equipment, the food, the housing, the transport, the medical care $\left.^{12} »\right)$. Le point qu'aborde ce personnage est concret, il est aussi réel dans notre monde que dans un monde envahi par les zombies. De plus, les questions financières sont au cœur de l'intrigue de World War $Z$ et agissent comme autant d'embrayeurs vers le réel.

17 Le titre complet World War $Z$ : an oral history of the zombie war transgresse aussi le cadre de la fiction: le pacte de lecture romanesque s'effrite à l'annonce de l'oralité qui soustendrait l'histoire narrée. Telle une légende transmise oralement, l'histoire qui va nous être contée s'inscrit dans un espace ambigu entre réalité et fiction. Elle a eu, ou aurait eu lieu, et se transmettrait désormais oralement. Elle résiste à devenir fiction. Ainsi, la série d'entretiens qui composent ce roman est précédée d'une « introduction », dans laquelle le 
narrateur pose le contexte dans lequel ont été réalisées ces interviews et qui s'achève sur ces mots :

Même si ce livre se compose essentiellement de souvenirs bruts, il inclut également nombre de données technologiques, sociales et économiques mentionnées dans le rapport original de la Commission [...]. Les questions qui jalonnent le texte ne font qu'anticiper celles que les lecteurs pourraient euxmêmes se poser. Je me suis efforcé d'éviter tout jugement et tout commentaire superflu, et s'il reste un «facteur humain », c'est surtout le mien $^{13}$.

Although this is primarily a book of memories, it includes many of the details, technological, social, economic, and so on, found in the original Commission report [...] Those questions included in the text are only there to illustrate those that might been posed by readers. I have attempt to reserve judgment, or commentary of any kind, and if there is a human factor that should be removed, let it be my own ${ }^{14}$.

Le dédoublement du lecteur, à la fois lecteur de la fiction écrite par Max Brooks-auteur et lecteur du rapport rédigé par Max Brooks-narrateur, contribue ici à brouiller la frontière du réel et du fictionnel, en réactivant et en actualisant le topos du manuscrit trouvé qui, selon Henri Coulet, " peut servir à créer une vraisemblance, ou au contraire (et en même temps) à qualifier une œuvre comme fictive ${ }^{15} »$. Dans le cas présent, il crée bien une vraisemblance, car le narrateur de l'introduction en a lui-même assuré la composition. Si World War $Z$ se configure comme un manuscrit oublié et trouvé, c'est que la compilation d'interviews constitue un rapport resté secret, que le narrateur choisit finalement de rendre public.

Enfin, les notes de bas de page qui jalonnent l'ensemble du roman de Max Brooks constituent un important indice de non-fictionnalité : qu'elles apportent des informations sur le contexte (par exemple concernant la chronologie des évènements : «On ignorait encore à l'époque si le virus pouvait survivre dans des déjections humaines ${ }^{16}$ "; « At that time, it was unsure wether the virus could survive in solid waste outside of the human body $\left.{ }^{17} »\right)$ ou qu'elles servent à éclairer le lecteur quant au sens d'un mot (comme c'est le cas avec le «Mkunga lalem : "l'anguille et l'épée", le premier art martial antizombies ${ }^{18}$ »; " Mkunga lalem (The Eel and the Sword), the world's premier antizombie martial art ${ }^{19}$. "), les notes de bas de page, en tant qu' " auxiliaires de lisibilitée ${ }^{20}$ ", contribuent à clarifier des éléments qui, se voyant prolongés d'une extension dans le péritexte, se trouvent chargés d'une part de réel. En effet, les notes impliquant la présence d'un auteur, elles suggèrent ici l'identité, la superposition de l'auteur et du narrateur, et opèrent un embrayage de la fiction au réel. De plus, la note de bas de page s'inscrit dans une marge qui est à la fois celle du texte et celle de la fiction : si elle peut donner une indication sur la fiction, la note de bas de page se situe concrètement hors de celle-ci. Ainsi, dans World War Z, Max Brooks multiplie les indices de non-fictionnalité et ce faisant, crée dans la fiction des interstices permettant au réel de s'y infiltrer.

\section{Effets de réel et série télévisée : The Walking Dead}

20 Ces zones de trouble en réel et fiction sont également présentes dans la série The Walking Dead. Il faut tout d'abord noter que la série télévisée est une forme spécifique qui construit, par sa durée et sa fréquence de diffusion, un rapport particulier au réel. Hervé 
Glevarec estime ainsi que " "l'effet de réel" caractéris[e] le régime fictionnel des séries télévisées contemporaines ${ }^{21} »$. Il explique notamment que

[1]'effet de réel de la série télévisée s'inscrit ici dans l'absence de téléologie connue à l'épisode visionné. Si aucune téléologie n'existe à la vision d'un épisode EOX, aucun "indice" ne peut être donc perçu par le téléspectateur puisqu'il n'y en a pas. L'absence de téléologie est ce qui rapproche les personnages des personnes, la fiction de la vie, à savoir que le vecteur rétrospectif-prospectif (de type pur hitchcockien) où le téléspectateur sait qu'Alfred Hitchcock connaît le coupable, est ici situé; que le téléspectateur sache ou non que le producteur ne sait pas laisse l'action fictionnelle, quoiqu'il en soit, dans une diégèse sans téléologie ${ }^{22}$.

21 La série télévisée se construisant en saisons dont le nombre est potentiellement illimité, la fin de ce type d'œuvre n'est pas l'enjeu de la diégèse. La particularité d'une série comme The Walking Dead est qu'elle présente une diégèse de la fin (du monde, de la civilisation humaine telle que nous la connaissons) dans une structure sérielle qui résiste à toute téléologie (du moins tant que Franck Darabont et Robert Kirkman n'auront pas annoncé l'arrêt de la production). La configuration a-téléologique de la série entre donc dans un rapport dialectique avec cette fiction de la fin qu'est l'histoire de zombie : la fin du monde s'étend et s'étire car tant qu'il y aura des hommes, il y aura des zombies; et l'apparition régulière de nouveaux personnages (le groupe principal étant amené à rencontrer d'autres groupes de survivants) ne laisse entrevoir qu'une fin du monde sans fin.

Tout autant que sa construction, le parti pris esthétique de la série renforce l'effet de réel : alors que les films de George A. Romero ${ }^{23}$, Lucio Fulci ${ }^{24}$ ou plus récemment Danny Boyle ${ }^{25}$ donnaient à voir l'exceptionnalité d'un monde en crise, jouant plus ou moins sur le spectaculaire ou le gore, une grande place est accordée à la vie quotidienne des survivants, à l'organisation sociale du groupe, aux questions concrètes d'hygiène, de logement, de nourriture, en particulier dans la première saison qui fait une large place aux questions domestiques. En effet, les zombies, appelés ici «rôdeurs" (walkers), sont une menace permanente que les survivants tentent tant bien que mal de juguler : cette menace perd donc de son exceptionnalité. Le quotidien des survivants devient ainsi la source essentielle des péripéties: faute de moyens de contraception, Lori tombe enceinte; les violences conjugales dont est victime Carol deviennent une histoire dans l'histoire, contribuant notamment à révéler le caractère violent de Shane qui moleste $\mathrm{Ed}$, le mari de cette dernière ; les problèmes conjugaux de Rick et Lori en viennent à devenir une intrigue de premier plan. Ces éléments ne sont pas en eux-mêmes des effets de réel, mais des composantes primordiales de la diégèse; en revanche, la structure narrative sérielle, en permettant une relation de long terme, hebdomadaire, avec le spectateur, en vient à faire des personnages des figures que le spectateur « fréquente » avec régularité.

La première saison de The Walking Dead, véritable saison d'exposition, installe ainsi un climat de familiarité grâce à la multiplication de référents communs aux personnages et aux téléspectateurs. Si la vie du camp de survivants est fréquemment troublée par des attaques de zombies, elle s'organise néanmoins progressivement en fonction de cet élément perturbateur qui devient lui-même quotidien, de sorte que cette aberration qu'est le mort-vivant intègre petit à petit la sphère des problèmes pragmatiques à prendre en compte. Par exemple, dans le quatrième épisode de la deuxième saison ${ }^{26}$, le groupe, hébergé dans la ferme d'Hershel Greene, trouve un zombie dans un puits et 
cherche le moyen de l'en faire sortir : non dénuée d'humour, cette péripétie n'a pourtant pas de nécessité ni de conséquence sur la trame principale : elle est présentée comme un désagrément quotidien parmi d'autres - un détail. Elle se configure dès lors comme un effet de réel - son apparente gratuité ne fait que renforcer le réalisme de l'ensemble. L'intégration progressive du zombie dans les tâches quotidiennes d'une collectivité permet en outre de mettre en place un savoir construit. Comment se débarrasser d'un zombie? Que faire en cas de morsure ? Comment agir en cas d'attaque?: ces questions font l'objet d'enseignement (dans la saison 1, Shane apprend aux membres du groupe à tirer; dans la saison 4, Carol enseigne aux enfants le maniement du couteau). Dans un monde plongé dans le chaos, les choses se ritualisent, donc se normalisent, et tendent ainsi à se rapprocher de l'univers socio-historique du spectateur.

Un autre élément de la diégèse concourt grandement à la construction d'indices de nonfictionnalité dans cette série télévisée: le fait que des personnages de premier plan puissent disparaître sans que la série ne s'achève, car la mort des héros augure, dans un long métrage à la construction plus classique, un dénouement proche. À ce propos, Hervé Glevarec note encore qu' ' il semble bien que les séries contemporaines aient introduit de façon nette la mort des personnages dans leur narration, transgressant ainsi la règle centrale de l'héroïsme de (toujours) sauver les personnages principaux ${ }^{27}$. » Ainsi, des personnages omniprésents à l'image depuis le début de la série meurent brutalement, et sont remplacés par d'autres protagonistes jusque-là secondaires: les morts de Shane (saison 2, épisode $12^{28}$ ), et de Lori (saison 3, épisode $4^{29}$ ), interviennent tout aussi brutalement que celle d'Otis (saison 2, épisode ${ }^{30}$ ), personnage qui n'intervient que dans un seul épisode. Il apparaît ainsi au spectateur qu'aucun personnage n'est à l'abri d'une mort brutale, qui ne marquera pas la fin de la série: le traitement de la mort renforce ainsi son absence de téléologie, donc son contact au réel.

Sur le plan axiologique, l'intégration des zombies à un univers familier induit une adhésion du spectateur à un système de valeurs a priori très éloigné du sien, en particulier dans les saisons 1 et $2^{31}$. Dans The Walking Dead, comme nous l'avons montré précédemment, la saison d'exposition met en place de manière récurrente des marques de référentialité qui rendent acceptable et cohérente la présence d'êtres aussi improbables que les morts-vivants. Acceptant leur présence, le spectateur accepte du même coup le système axiologique et éthique qui les encadre : tuer peut être nécessaire, y compris tuer des vivants; la défense de l'abri prime sur la solidarité humaine. Dans l'épisode 8 de la $2^{\mathrm{e}}$ saison $^{32}$, Rick rencontre deux survivants qui le menacent d'une arme pour obtenir l'adresse de la ferme d'Hershel. Rick les abat: le spectateur est forcé d'adhérer à cette violence, devenue nécessaire et donc acceptable. Se produit ainsi une sorte de prolifération de l'effet de réel: pris dans une structure narrative sérielle, donc récurrente, qui multiplie les effets de réel, le spectateur n'attend plus que la série se rapproche de son système de valeur, mais adhère à celui que la fiction lui propose. En un sens, les indices de non-fictionnalité permettent à la fiction d'agir sur le spectateur.

La production d'effets de réel ou indices de non-fictionnalité, dans les romans de Max Brooks comme dans la série The Walking Dead, induit une remotivation de la figure du mort-vivant, qui n'incarne plus une crise ou une apocalypse, mais devient un contexte, un accident vraisemblable. On observe ainsi le passage d'une figure du zombie comme mythe, comme figure apocalyptique, à une modernité du zombie, qui n'est plus figure de la fin mais figure sans fin, qui est vouée à se répéter (The Zombie Survival Guide de Max Brooks dresse une liste des épidémies recensées et vise à prévenir les épidémies à venir) 
ou à perdurer comme contexte (la forme sérielle de The Walking Dead tend bien à inscrire les attaques de morts-vivants dans une structure de répétition). Le mort-vivant, figure aberrante qui transgresse les lois de la nature, passe ainsi de l'accident à la permanence, révélant une acceptation croissante de la violence : cette mutation de la figure du mortvivant laisse alors entrevoir un effondrement de la civilisation désormais devenu vraisemblable.

Cette multiplication des effets de réel non seulement renforce l'immersion du lecteurspectateur dans la fiction, mais plus encore l'amène à adhérer aux règles de ce monde chaotique pourtant aux antipodes du réel. Aussi le désir de voir le monde tel qu'on le connaît disparaitre est-il plus que jamais mobilisé dans ces fictions. Max Brooks y voit même un exutoire aux vicissitudes d'une société profondément insécurisante. Il déclare ainsi: «I think zombies are safe. Zombies are manageable. You can't shoot the Gulf oil spill in the head. [...] What does the global financial meltdown of 2008 mean? I can't explain it, and I sure know you can't shoot it in the head ${ }^{33}$.» («Je pense que les zombies sont sûrs. Ils sont gérables. Vous ne pouvez pas tirer dans la tête de la marée noire du Golfe du Mexique. [...] Que signifie la crise financière mondiale de 2008 ? Je ne peux pas l'expliquer, et je suis certain que je ne peux pas lui tirer dans la tête. »). Dans ces fictions poreuses au réel, le zombie serait alors un ennemi identifiable, d'origine humaine, et donc paradoxalement rassurant, car toujours destructible: un dérivatif face au sentiment de perte de contrôle qui anime les sociétés postindustrielles.

\section{BIBLIOGRAPHIE}

BARTHES, Roland, «L'effet de réel », Communications, nº11, 1968, pp. 84-89.

BETAN, Julien et COLSON, Raphaël, Zombies!, nouvelle édition augmentée, Les moutons électriques, 2013.

BROOKS, Max, Zombie Survival Guide, Three rivers Press, New York, 2003 ; Guide de survie en territoire zombie, traduction de Patrick Imbert, Calmann-Lévy, coll. Le Livre de Poche, 2009.

-World War Z. An oral history of the zombie war, Broadway Paperbacks, New York, 2006; World War Z, traduction de Patrick Imbert, Calmann Lévy, coll. Le Livre de Poche, 2009.

COULET Henri, «Le topos », in Pierre Rodriguez et Michèle Weil (dir.), Vers un thésaurus informatisé : topique des ouvertures narratives avant 1800. Actes du Quatrième Colloque international SATOR, PULM-Université Paul-Valéry, Montpellier, 1991, p. 330.

COULOMBE, Maxime, Petite philosophie du zombie, PUF, 2012.

GLEVAREC, Hervé, « Trouble dans la fiction. Effets de réel dans les séries télévisées contemporaines et post-télévision », Questions de communication n¹8, 2010, p. 215-238.

MILHE POUTINGON, Gérard, « Les notes marginales dans le Champfleury de Geoffroy Tory : des auxiliaires de lisibilité », in DÜRENMATT, Jacques et PFERSMANN, Andreas (dir.), L'Espace de la note, La Licorne, 2004, p 67 
SAINT-GELAIS Richard, «L'effet de non-fiction : fragments d'une enquête », L'effet de fiction, colloque en ligne Fabula, 2001, URL : http://www.fabula.org/anciens_colloques/effet/ interventions/16.php, consulté le 22 novembre 2013.

SCHAEFFER, Jean-Marie, Pourquoi la fiction ?, Seuil, 1999.

TOWNSEND, Allie, « "I'm Just A Zombie Nerd” The Max Brooks Interview, Part One », Time Magazine, juillet 2010, URL : http://techland.time.com/2010/07/15/im-just-a-zombie-nerd-themax-brooks-interview-part-one/\#ixzz216gDdTk4, consulté le 22 novembre 2013.

«"It's Sense \& Sensibility \& Sleestacks Now” The Max Brooks Interview, Part Two » Time Magazine, juillet 2010, URL: http://techland.time.com/2010/07/20/were-doing-sensesensibility-sleestacks-now-the-max-brooks-interview-part-two/\#ixzz216hvuJsZ, consulté le 22 novembre 2013.

THORET, Jean-Baptiste (dir.), Politique des zombies. L'Amérique selon George A. Romero, Ellipses, 2007.

VUCKOVIC, Jovanka, Zombies! Une histoire illustrée des morts-vivants, préface Georges A. Romero, Hoëbecke, 2011.

Filmographie

BOYLE Danny, 28 days later, 2002; 28 weeks later, 2007.

DARABONT Frank et KIRKMAN Robert, The Walking Dead, saison 1, 2010; saison 2, 2011; saison 3, 2012; saison 4, 2013.

FULCI, Lucio, Zombi 2, 1979 ; E tu vivrai nel terrore - L'aldilà, 1981, Zombi 3, 1988.

ROMERO, George A., Night of the Living Dead, 1968; Dawn of the Dead, 1978; Land of the Dead, 2005; Diary of the Dead, 2008; Survival of the Dead, 2010.

\section{NOTES}

1. BETAN, Julien et COLSON, Raphaël, Zombies!, nouvelle édition augmentée, Les moutons électriques, 2013, p. 4.

2. COULOMBE, Maxime, Petite philosophie du zombie, PUF, 2012, p. 17.

3. Ibid., p. 18.

4. THORET, Jean-Baptiste, «Ils sont comme nous », in THORET, Jean-Baptiste (dir.), Politique des zombies. L'Amérique selon George A. Romero, Ellipses, 2007, p. 13.

5. SCHAEFFER, Jean-Marie, Pourquoi la fiction?, Seuil, 1999, p. 202.

6. BARTHES, Roland, «L'effet de réel », Communications, $n^{\circ} 11,1968$, p. 88.

7. SAINT-GELAIS, Richard, "L'effet de non-fiction : fragments d'une enquête ", L'effet de fiction, colloque en ligne, Fabula, 2001, URL: http://www.fabula.org/anciens_colloques/effet/ interventions/16.php, consulté le 22 novembre 2013.

8. Ibid.

9. TOWNSEND, Allie, " "It's Sense \& Sensibility \& Sleestacks Now" The Max Brooks Interview, Part Two », Time Magazine, juillet 2010, URL: http:// techland.time.com/2010/07/20/were-doing-sense-sensibility-sleestacks-now-the-maxbrooks-interview-part-two/\#ixzz2l6hvuJsZ, consulté le 10 décembre 2013. Nous traduisons.

10. BOYLE, Danny (réalisateur), 28 days later, Royaume-Uni, 2002. 
11. BROOKS, Max, World War Z, traduction de Patrick Imbert, Calmann Lévy, coll. Le Livre de Poche, 2009, p. 88.

12. BROOKS, Max, World War Z. An oral history of the zombie war, Broadway Paperbacks, New York, 2006 p. 66-67.

13. BROOKS, Max, World War Z, op.cit., p. 12.

14. BROOKS, Max, World War Z, op.cit., p. 3.

15. COULET Henri, «Le topos ", in RODRIGUEZ, Pierre et WEIL, Michèle (dir.), Vers un thésaurus informatisé: topique des ouvertures narratives avant 1800. Actes du Quatrième Colloque international SATOR, Imprimerie de recherche-Université Paul-Valéry, Montpellier, 1991, p. 330 .

16. BROOKS, Max, World War Z, op.cit., p. 71.

17. BROOKS, Max, World War Z, op.cit., p. 53.

18. BROOKS, Max, World War Z, op.cit., p. 410.

19. BROOKS, Max, World War Z, op.cit., p. 326.

20. MILHE POUTINGON, Gérard, «Les notes marginales dans le Champfleury de Geoffroy Tory : des auxiliaires de lisibilité ", in DÜRENMATT, Jacques et PFERSMANN, Andreas (dir.), L'Espace de la note, La Licorne, 2004, p. 67.

21. GLEVAREC, Hervé, "Trouble dans la fiction. Effets de réel dans les séries télévisées contemporaines et post-télévision ", Questions de communication n¹8, 2010, p. 229.

22. Ibid. , p. 229.

23. ROMERO, George A., Night of the living dead, 1968; Dawn of the dead, 1978; Day of the Dead, 1985; Land of the dead, 2005; Diary of the Dead, 2008; Survival of the Dead, 2010.

24. FULCI, Lucio, Zombi 2, 1979 ; E tu vivrai nel terrore - L'aldilà, 1981 ; Zombi 3, 1988.

25. BOYLE, Danny, 28 days later, 2002; 28 weeks later, 2007.

26. The Walking Dead, saison 2, épisode 4, "Cherokee Rose », scénario Evan Reilly, réalisation Billy Gierhart, première diffusion aux États-Unis sur AMC le 6 novembre 2011.

27. GLEVAREC, Hervé, op. cit., p. 231.

28. The Walking Dead, saison 2, épisode 8, «Better Angels ", scénario Evan Reilly et Glen Mazzara, réalisation Guy Ferland, première diffusion aux États-Unis sur AMC le 11 mars 2012.

29. The Walking Dead, saison 2, épisode 8, « Killer Within », scénario Sang Kyu Kim, réalisation Guy Ferland, première diffusion aux États-Unis sur AMC le 4 novembre 2012.

30. The Walking Dead, saison 2, épisode 2, «Bloodletting», scénario Glen Mazzara, réalisation Ernest R. Dickerson, première diffusion aux États-Unis sur AMC le 23 octobre 2011.

31. En effet, à partir de la saison 3 , une réflexion éthique se met progressivement en place : la banalisation de la violence fait l'objet de nombreux questionnements. Ils sont notamment incarnés par le personnage de Rick qui, voyant son jeune fils Carl adopter une attitude de plus en plus détachée face à la violence, s'interroge sur le système de valeur qui se met en place dans ce monde dévasté.

32. The Walking Dead, saison 2, épisode 8, «Nebraska ", scénario Evan Reilly, réalisation Clark Johnson, première diffusion aux États-Unis sur AMC le 12 février 2012.

33. TOWNSEND, Allie, "It's Sense \& Sensibility \& Sleestacks Now" The Max Brooks Interview, Part Two", Time Magazine, juillet 2010, URL: http:// techland.time.com/2010/07/20/were-doing-sense-sensibility-sleestacks-now-the-maxbrooks-interview-part-two/\#ixzz216hvuJsZ, consulté le 10 décembre 2013. Nous traduisons. 


\section{RÉSUMÉS}

La figure du mort-vivant s'est développée dans le cinéma comme un motif qui catalyse les angoisses occidentales et qui révèle un désir d'assister à l'Apocalypse. Le zombie est par définition une figure aberrante, impossible, transgressant à la fois les lois de la nature et celle de l'humain. Mais depuis les années 2000, cette figure connaît une mutation, sous la plume du romancier américain Max Brooks (The Zombie Survival Guide, 2003 et World War Z, 2006) et dans la série télévisée de Frank Darabont et Robert Kirkman The Walking Dead: en effet, ces œuvres mettent en place de manière récurrente des effets de réel qui amènent à faire évoluer considérablement les codes du genre. Nous verrons ainsi comment, d'une figure fantasmatique de la fin du monde, le zombie devient paradoxalement le moyen de faire entrer le réel dans la fiction.

The living-dead persona has grown in cinema by catalyzing western fears and revealing a wish to attend the Apocalypse. By definition, zombie is an aberrant, impossible character, transgressing both natural and human laws. But since the 2000's, this character has mutated, particularly in Max Brooks' novels (The Zombie Survival Guide, 2003 and World War Z, 2006) and in the Frank Darabont and Robert Kirkman's TV serie The Walking Dead: indeed, these works set recurrent "effects of reality" that have triggered an important evolution of the zombie genre. This article proposes to examine how the zombie, from a fantasmatic figure of the end of the world, paradoxically becomes a way to bring reality into fiction.

\section{AUTEUR}

\section{CLÉMENTINE HOUGUE}

Docteur en Littérature Générale et Comparée, Clémentine Hougue a soutenu en 2012, sous la direction de Jean Bessière, une thèse intitulée : « Le cut-up. Ses antécédents, ses développements, en Europe et aux États-Unis au XX $X^{\mathrm{e}}$ siècle ». Elle a publié plusieurs articles sur William S.

Burroughs, le cut-up et le collage. Ses recherches portent également sur les avant-gardes, la culture de masse et les liens entre littérature, arts et politique. 